RHINOLOGY

\title{
Clinical characteristics of patients with granulomatosis with polyangiitis and microscopic polyangiitis in ENT practice: a comparative analysis
}

\author{
Caratteristiche cliniche dei pazienti con granulomatosi con poliangioite \\ e poliangioite microscopica nella pratica clinica ORL: analisi comparativa
}

\author{
J. WOJCIECHOWSKA, T. KRĘCICKI \\ Department and Clinic of Otolaryngology, Head and Neck Surgery, Wrocław Medical University Hospital, Borowska, Poland
}

\section{SUMMARY}

ENT manifestations are commonly observed in patients with small vessel vasculitis (SVV). The main aim of this study was to analyse and present the clinicopathological characteristics of individuals with SVV emphasising otorhinolaryngological symptoms. This study evaluated 64 patients, 41 with granulomatosis with polyangiitis (GPA) and 23 with microscopic polyangiitis (MPA). Herein, we compare the clinicopathologic features of GPA and MPA. The average age at diagnosis was 50.2 and 56.2 years, for GPA and MPA, respectively. 57 patients (89\%) were antineutrophil cytoplasmic antibody (ANCA) positive, 34 (59.6\%) for anti-proteinase 3 (PR3)-ANCA and 21 (36.8\%) for myeloperoxidase (MPO)-ANCA. 7 patients (10.9\%) were ANCA negative. The most commonly affected organs were lungs (76.56\%), ear, nose, throat (ENT) (75\%) and kidneys (73.44\%). ENT disorders mainly appeared as chronic rhinosinusitis and epistaxis and preceded SVV diagnosis by an average 14.4 months. In the majority of patients, ENT disorders were the first symptoms of SVV and preceded its systemic transformation. Pulmonary, ENT and nervous manifestations were more common in GPA, whereas the prevalence of renal, gastrointestinal, cutaneous, cardiovascular and ocular disorders was higher in MPA. The results of our study emphasise the high prevalence of ENT symptoms in patients with SVV, especially in those with GPA. We highlight the significant role of the otorhinolaryngologist in early SVV diagnosis and management. Any patient with persistent ENT symptoms or ENT dysfunctions not responding to standard otorhinolaryngological treatment should be precisely and rapidly evaluated for the presence of systemic dysfunctions (especially renal and pulmonary). Realising the differences and similarities between GPA and MPA is crucial in undelayed SVV diagnosis and proper treatment.

KEY WORDS: Granulomatosis with polyangiitis • Microscopic polyangiitis • Vasculitis • MPO-ANCA • PR3-ANCA

\section{RIASSUNTO}

Nei pazienti con vasculiti dei piccoli vasi (SVV), si osservano frequentemente manifestazioni del distretto otorinolaringoiatrico (ORL). Lo scopo principale di questo studio è stato quello di analizzare e presentare le caratteristiche clinicopatologiche degli individui con SVV, enfatizzando i sintomi della sfera ORL. Sono stati valutati 64 pazienti, 41 con granulomatosi con poliangioite (GPA) e 23 con poliangioite microscopica (MPA). Abbiamo presentato e paragonato le manifestazioni clinicopatologiche di GPA e MPA. L'età media alla diagnosi è stata rispettivamente di 50,2 e 56,2 anni per GPA e MPA. 57 pazienti (89\%) erano positivi per gli anticorpi anti citoplasma dei neutrofili (ANCA), 34 (59,6\%) per gli anti proteinasi 3-ANCA e 21 (36,8\%) per mieloperossidasi (MPO)-ANCA. 7 pazienti erano ANCA negativi. Gli organi più frequentemente colpiti sono stati polmone (76,5\%), ORL (75\%) e reni (73,4\%). Le problematiche ORL si sono manifestate più frequentemente sotto forma di rinosinusite cronica ed epistassi ed hanno preceduto in media la diagnosi di SVV di circa 14,4 mesi. Nella maggior parte dei pazienti i disturbi ORL hanno rappresentato il primo sintomo di SVV, precedendone la trasformazione sistemica. Nella GPA, sono risultate più frequenti le manifestazioni polmonari, ORL e del sistema nervoso, mentre nella MPA quelle renali, gastrointestinali, cutanee, cardiovascolari ed oculari. I risultati del nostro studio hanno enfatizzato l'alta prevalenza di sintomi ORL nei pazienti con SVV, specialmente in quelli con GPA. Vogliamo quindi sottolineare il ruolo importante dello specialista ORL nella diagnosi precoce e nel conseguente precoce trattamento delle SVV. Ogni paziente con sintomi o disfunzioni ORL persistenti e non responsivi alle terapie standard, deve essere valutato per la presenza di manifestazioni sistemiche (specialmente renali e polmonari). Evidenziare le differenze e le somiglianze tra GPA e MPA è cruciale per una precoce diagnosi di SVV e per iniziare una terapia adeguata.

PAROLE CHIAVE: Granulomatosi con polyangioite $\bullet$ Poliangioite microscopica $\bullet$ Vasculiti $\bullet$ MPO-ANCA $\bullet P R 3-A N C A$

Cite this article as: Acta Otorhinolaryngol Ital 2018;38:517-527. https://doi.org/10.14639/0392-100X-1776

(C) Società Italiana di Otorinolaringoiatria e Chirurgia Cervico-Facciale 


\section{Introduction}

Small vessel vasculitis (SVV) is a heterogeneous group of idiopathic disorders characterised by necrosis and inflammation of small-sized blood vessels ${ }^{1}$. SVV with the serum presence of antineutrophil cytoplasmic antibody (ANCA) is classified as ANCA-associated vasculitis (AAV). The main variants of AAV are granulomatosis with polyangiitis (GPA; previously Wegener's granulomatosis), eosinophilic granulomatosis with polyangiitis (EGPA) and microscopic polyangiitis (MPA), including renal-limited vasculitis (RLV) ${ }^{2}$. The presence of ANCA in sera constitutes a crucial serologic marker of AAV, even if there are cases with negative ANCA. ANCA patterns are classified as anti-proteinase 3 (PR3) - ANCA (cytoplasmic anti-neutrophil cytoplasmic antibody, cANCA), and anti-myeloperoxidase (MPO) - ANCA (perinuclear- anti-neutrophil cytoplasmic antibody, pANCA) ${ }^{3}$. The majority of cases with ANCA negativity is observed in patients in early stages of the disease or in those with localised disease that is limited to organs of the upper respiratory tract and lungs ${ }^{45}$. Besides the upper respiratory tract, AAV typically affects the lower respiratory tract and kidneys. Nevertheless, any organ may be involved. In the great majority of patients with AAV, especially in those with GPA, the initial symptoms of the disease are otorhinolaryngological abnormalities ${ }^{67}$. In MPA, involvement of head and neck organs is less common. Besides the fact that exact diagnosis is based on the combination of particular serologic, laboratory, imaging and histopathologic features, clinical manifestations are of great value in early disease recognition and therapy ${ }^{8}$. It has been suggested that Staphylococcus aureus (S. aureus) may act as a molecular mimicry for proteinase 3 (PR3) and may be a trigger point of PR3 autoimmunity and PR3-ANCA autoantibody production. It was observed that the nasal carriage of $S$. aureus in patients during GPA remission predisposes to disease relapses. This explains the validity of anti-staphylococcal prophylaxis with trimethoprim/sulfamethoxazole in patients with remission ${ }^{29}$. The question of whether or not there is a possible role for $S$. aureus in MPA pathogenesis still remains unanswered.

In this study, we present the clinicopathological features of a group of our patients with AAV. We report in detail the particular manifestations in subjects with GPA and MPA, comparing these two groups. According to the high frequency of ENT disorders in patients with AAV, we highlight the importance of the otorhinolaryngologist in establishing AAV diagnosis and its management.

\section{Materials and methods}

\section{Clinical data}

An institutional ethics committee approved the study, and the study protocol complied with the Helsinki Declaration. We examined 64 patients with SVV who were admitted to our tertiary referral university hospital between January 2016 and April 2017. Patients' medical SVV-related records gathered before January 2016 were included in the study. The American College of Rheumatology (ACR) classification criteria for vasculitis, and the Chapel Hill Consensus Conference (CHCC) classification were used to assign patients to particular subgroups as GPA or MPA, respectively ${ }^{1011}$. According to these classifications, 41 patients met the criteria for GPA and 23 patients were classified as suffering from MPA.

Demographic features included gender, age and age at diagnosis. Clinical manifestations and laboratory investigation were examined in all patients at the time of hospitalisation. Clinical assessment was performed by an otolaryngologist (JW) and nephrologist (MK) to minimise bias. Laboratory investigation comprised presence of ANCA, complete blood count (CBC), serum creatinine levels, serum urea levels, urinalysis, C-reactive protein (CRP), erythrocyte sedimentation rate (ESR) and procalcitonin (PCT) levels. All laboratory tests were performed at the same hospital laboratory.

Measurement of serum ANCA levels was done using indirect immunofluorescence (IFA). In ANCA-positive patients detected by IFA, enzyme-linked immunosorbent assay (ELISA) was used to determine the serum levels of PR3-ANCA and MPO-ANCA. ANCA assessment was done at least twice, in time intervals, to eliminate possible false negative or false positive results.

When available, nasal swabs for bacterial pathogens dwelling in the nasal cavity were examined retrospectively.

Clinical evaluation of systemic involvement was enriched with imaging studies (CT scan) of the thoracic cavity.

\section{Otorhinolaryngological examination}

Otorhinolaryngological investigation was performed by an otorhinolaryngologist (JW) and was based on standard clinical examination consequently supported by endoscopic evaluation (flexible and rigid Karl Storz endoscopes) in patients with positive or uncertain findings. Indirect laryngoscopy (Karl Storz laryngoscope) was used in all patients to assess the oropharynx, larynx and hypopharynx. Audiology tests including pure tone audiometry (PTA) and impedance audiometry (IA) were used 
in patients with hearing deficits detected in traditional voice tests (TVT) and in tuning fork test (TFT). Computed tomography (CT) of the sinuses was performed in individuals with sinonasal abnormalities found in ENT examination and in those with positive history of sinonasal manifestations.

\section{Histology}

Histologic data were obtained from 48 biopsies taken from 42 patients. Five patients with clinical presentation strongly suggesting AAV were biopsied more than once because of nondiagnostic histologic results. Twenty-two patients did not have any biopsy. Four subjects underwent histologic examination of more than one organ.

It was established that biopsy presenting necrotising vasculitis confirmed the presence of AAV. Histologic findings in our patients were classified as positive or negative for particular subgroups of AVV according to standards, i.e. small-vessel granulomatous necrotising vasculitis was classified as GPA, and small-vessel nongranulomatous necrotising vasculitis was classified as MPA ${ }^{2}$.

\section{Disease severity}

Disease severity stages of enrolled patients were assessed using European Vasculitis Study Group (EUVAS) recommendations ${ }^{12}$. Patients were classified as suffering from localised, early systemic, generalised or severe AAV. A localised form of AAV was characterised by ear, nose, throat (ENT) or lung involvement with serum creatinine levels < $120 \mu \mathrm{mol} / \mathrm{l}(1.3 \mathrm{mg} / \mathrm{dl})$; an early systemic form was defined as ENT and pulmonary involvement in combination with involvement of an organ outside the upper respiratory tract and serum creatinine levels $<120 \mu \mathrm{mol} / \mathrm{l}$ $(1.3 \mathrm{mg} / \mathrm{dl})$. Generalised forms included cases with vasculitis in organs outside the ENT and lungs, threatened function of vital organ and serum creatinine levels $<500$ $\mu \mathrm{mol} / \mathrm{l}(5.5 \mathrm{mg} / \mathrm{dl})$. A severe form was defined as vasculitis in organs outside the ENT and lungs, failure of vital organ function and serum creatinine levels $>500 \mu \mathrm{mol} / 1$ $(5.5 \mathrm{mg} / \mathrm{dl})$.

\section{Results}

In total, 64 patients were enrolled in our study. We identified 41 individuals (19 males/22 females) with GPA and 23 individuals (10 males/ 13 females) with MPA. The mean age of patients during hospitalisation in our hospital was 54 years (55.3 years in males/53.1 years in females) for GPA, and 60.3 years (55.9 years in males/63.6 years in females) for MPA, respectively. The average age at the time of AAV diagnosis was 50.2 years and 56.2 years, for GPA and MPA, respectively. In females, the mean age at diagnosis reached 53 years, whereas in men it was 55.3 years (Table I). In the majority of cases the initial symptoms were ENT disorders. The average duration between first ENT disorders and AAV diagnosis was 14.5 months, ranging between 1 month to 36 months. The most common initial ENT manifestations were recurrent epistaxis and persistent chronic rhinosinusitis not responding to standard treatment. Less frequent complaints at disease onset were chronic purulent otitis media and sudden sensorineural hearing loss.

\section{Serology}

The majority of patients, 57 patients $(89.1 \%)$, were ANCA positive, among which 34 (59.6\%) were positive for PR3-ANCA and 21 (36.8\%) for MPO-ANCA. Two patients $(3.5 \%)$ were positive for both PR3-ANCA and MPO-ANCA. Thirty-six patients $(100 \%)$ positive for PR3-ANCA were classified as suffering from GPA. 21 patients $(91.3 \%)$ positive for MPO-ANCA were classified as suffering from MPA. Seven patients (10.9\%) were identified as ANCA negative (negative serum PR3-ANCA and MPO-ANCA levels) and 5 individuals with GPA and 2 with MPA, respectively.

\section{Other biochemical findings}

The most common biochemical findings in our patients with GPA were neutrophilia in CBC, microscopic and gross haematuria by urinalysis, and elevated ESR (Table II). In the MPA cohort, the most common abnormalities were neutrophilia, microscopic haematuria, elevated ESR and elevated serum creatinine and urea.

Normal levels of CRP did not exceed $5 \mathrm{mg} / \mathrm{l}$. We classified CRP as elevated when it reached more than $10 \mathrm{mg} / \mathrm{l}$. In our cohort, CRP was increased in 35 subjects (GPA/MPA, 23/13). ESR in normal conditions should not surpass 20 $\mathrm{mm} / \mathrm{h}$, depending on age and gender. We categorised ESR as elevated when it exceeded $30 \mathrm{~mm} / \mathrm{h} .48$ patients (GPA/ MPA, 31/17) in our cohort presented abnormally high ESR levels. PCT was elevated $(>0.5 \mathrm{ng} / \mathrm{ml})$ in 25 individuals (GPA/MPA, 17/8).

Serum creatinine levels above normal level ( $>1.3 \mathrm{mg} / \mathrm{dL})$ was reported in 41 cases (GPA/MPA, 24/17), whereas serum urea levels were increased $(>40 \mathrm{mg} / \mathrm{dL})$ in 41 patients (GPA/MPA, 23/18).

According to ANCA antibody status, patients with the presence of MPO-ANCA antibodies were more prone to have elevated serum creatinine and urea levels than patients with PR3-ANCA antibodies. A similar observation has also been reported by other authors ${ }^{13}{ }^{14}$.

Abnormal urinalysis was reported in 49 patients (GPA/ 
Table I. Characteristics of patients with GPA and MPA.

\begin{tabular}{|c|c|c|}
\hline & $\mathrm{GPA}, \mathrm{n}=41$ & MPA, $n=23$ \\
\hline Gender (male/female) \% & $19 / 22(46.3 / 53.7)$ & $10 / 13(43.5 / 56.5)$ \\
\hline Mean age (male/female) in years & $54(55.3 / 53.1)$ (range 20-80) & $60.3(55.9 / 63.6)$ (range 35-85) \\
\hline Mean age at diagnosis (male/female) in years & 50.2 & 56.2 \\
\hline PR3-ANCA positivity (+), n (\%) & $34(82.9)$ & $0(0 \%)$ \\
\hline MPO-ANCA positivity (+), n (\%) & $0(0 \%)$ & $21(91.3 \%)$ \\
\hline PR3-ANCA(+) and MPO-ANCA(+), n (\%) & $2(4.88 \%)$ & $0(0 \%)$ \\
\hline ANCA negativity (-), n (\%) & $5(12.2 \%)$ & $2(8.7 \%)$ \\
\hline Colonisation of Staphylococcus aureus, n (\%) & $12(29.3 \%)$ & $2(8.7 \%)$ \\
\hline Histopathologic confirmation, n (\%) & $32(78 \%)$ & $11(47.8 \%)$ \\
\hline \multicolumn{3}{|l|}{ Disease severity stage: } \\
\hline Localised, n (\%) & $13(31.7 \%)$ & $2(8.7 \%)$ \\
\hline Early systemic, n (\%) & $2(4.88 \%)$ & $2(8.7 \%)$ \\
\hline Generalised, n (\%) & $18(43.9 \%$ & $10(43.48 \%)$ \\
\hline Severe, $\mathrm{n}(\%)$ & $8(19.5 \%)$ & $9(39.1 \%)$ \\
\hline
\end{tabular}

MPA, 29/20). In our cohort, patients with MPA were more prone to present disorders in urinalysis. The most frequent urinalysis finding was the concomitant presence of proteinuria, microscopic haematuria, gross haematuria and leukocyturia.

The prevalence of $\mathrm{CBC}$ disorders was higher in subjects with MPA than in those with GPA. Abnormalities in CBC were detected in 59 patients (GPA/MPA, 37/22). The most common findings in CBC were leukocytosis, neutrophilia, lymphopenia and anaemia. Thrombocytosis was less frequent and was reported in only 9 cases. ANCA negative patients typically presented concomitant leukocytosis, neutrophilia and lymphopenia. Laboratory findings are presented in Table II.

\section{Microbiology}

The majority of patients with GPA, and only two patients with MPA, were colonised by $S$. aureus. The most common staphylococcal nidus was the nasal cavity.

In our study, 37 patients (57.8\%) underwent microbiological examination among which we identified 14 individuals (37.8\%) colonised by S. aureus, 12 patients $(85.7 \%)$ with GPA and $2(14.3 \%)$ with MPA. The majority of infected patients was PR3-ANCA positive and presented a localised form of the disease. Nasal carriage of $S$. aureus was the most frequently colonised area, and was established in 12 patients $(85.7 \%)$ in which 2 were ANCA negative. Nasal carriage of $S$. aureus was identified only in one patient with MPA. Other pathogens identified in our patients were Fussarium (1 patient; 2.7\%), Acinetobacter baumanii (2 patients; 5.4\%), Enterobacter cloacae (1 patient; 2.7\%), Escherichia coli (2 patients; 5.4\%), Klebsiella pneumo-
Table II. Laboratory findings in patients with AAV.

\begin{tabular}{lcc} 
Laboratory findings, $\mathbf{n}(\%)$ & GPA, $\mathbf{n}=\mathbf{4 1}$ & MPA, $\mathbf{n}=\mathbf{2 3}$ \\
CRP $(>10 \mathrm{mg} / \mathrm{l})$ & $23(56)$ & $13(56.5)$ \\
ESR $(>30 \mathrm{~mm} / \mathrm{h})$ & $31(75.6)$ & $17(73.9)$ \\
PCT $(>0,5 \mathrm{ng} / \mathrm{ml})$ & $17(41.46)$ & $8(34.78)$ \\
Serum creatinine $(>1.3 \mathrm{mg} / \mathrm{dl})$ & $24(58.5)$ & $17(73.9)$ \\
Serum urea $(>40 \mathrm{mg} / \mathrm{dL})$ & $23(56.1)$ & $18(78.3)$ \\
Urinalysis - abnormalities: & $29(70.7)$ & $20(87)$ \\
Proteinuria & $21(51.2)$ & $16(69.6)$ \\
Microscopic haematuria & $24(58.5)$ & $19(82.6)$ \\
Gross haematuria & $24(58.5)$ & $15(65.2)$ \\
Leukocyturia & $17(41.5)$ & $13(56.5)$ \\
CBC - abnormalities: & $37(90.2)$ & $22(95.7)$ \\
Leukocytosis & $24(58.5)$ & $13(56.5)$ \\
Neutrophilia & $31(75.6)$ & $19(82.6)$ \\
Lymphopenia & $24(58.5)$ & $16(69.6)$ \\
Anaemia & $24(58.5)$ & $16(69.6)$ \\
Thrombocytosis & $8(19.5)$ & $1(4.3)$ \\
\hline
\end{tabular}

CRP: C-reactive protein; ESR: Erythrocyte sedimentation rate; CBC: Complete blood count; PCT: Procalcitonin.

niae (2 patients; 5.4\%), Pseudomonas aeruginosa (2 patients; 5.4\%) and Enterecoccus faecium (1 patient; 2.7\%).

\section{Histology}

Among 48 biopsies taken from 42 patients, 43 (89.6\%) met the criteria for AAV. Thirty-two biopsies (74.4\%) were classified as typical for GPA, and $11(25.6 \%)$ suggested MPA. The AAV-positive biopsies were taken from kidney $(n=21)$, nose $(n=11)$, ear $(n=4)$, trachea $(n=1)$, lung $(n=1)$, muscle $(n=1)$, skin $(n=1)$, auto-amputated 
finger $(n=1)$, sphenoid sinus $(n=1)$ and spleen $(n=1) .3$ biopsies taken from nasal cavity and 2 taken from kidney were not diagnostic for any type of AAV.

\section{Radiologic imaging of the thoracic cavity}

CT scan of the thoracic cavity was performed in the majority of patients. We identified various vasculitis-related chest abnormalities in 46 patients $(71.88 \%), 30$ patients $(73.17 \%)$ with GPA and $16(69.6 \%)$ with MPA. The most common radiologic findings were pulmonary nodules $(34.38 \%)$, diffuse ground-glass opacity (GGO) - a result of diffuse alveolar haemorrhage [DAH] (23.44\%), and inflammatory infiltrations $(45.3 \%)$, respectively. Interstitial fibrosis or thickening were rarer $(17.19 \%$ of patients), whereas pneumothorax presented only in one patient $(1.56 \%)$. Pleural effusion was observed in 6 patients $(9.38 \%)$. In summary, pulmonary nodules, GGO, inflammatory infiltrations, pneumothorax and pleural effusion were more common in those suffering from GPA. In contrast, interstitial fibrosis or thickening was observed more often in patients with MPA.

\section{Disease severity}

The most common disease stages in our cohort were generalised AAV, followed by severe form, and they were more often observed in patients with MPA than in those with GPA. The majority of localised forms of AAV was reported in subjects with GPA.

In our study, 15 patients (23.44\%), 13 vs. 2, for GPA and MPA, respectively, met the criteria for localised disease. In the group of 13 patients with a localised form, 7 subjects were PR3-ANCA positive, 4 subjects were ANCA negative and 2 individuals were positive for both PR3ANCA and MPO-ANCA. All patients with localised MPA were positive for MPO-ANCA. 4 patients (6.25\%), 2 individuals with GPA and 2 with MPA, were classified as suffering from early systemic disease. 28 people $(43.75 \%)$ presented a generalised form, 18 with GPA and 10 with MPA. 17 patients (26.56\%), 8 vs. 9, for GPA and MPA, respectively, developed severe AAV (Table I).

\section{Clinical manifestations (Table III)}

\section{ENT involvement}

In general, 48 patients (75\%), 35 patients $(85.37 \%)$ with GPA and 13 patients (56.52\%) with MPA, presented ENT involvement (Table IV).

Nose and paranasal sinuses. The most commonly affected ENT organs in patients with AAV were paranasal sinuses. In our study, subjects with GPA were more prone to suffer from sinonasal disorders than those with MPA.
In total, 45 patients $(70.3 \%)$ presented vasculitis-related abnormalities in nose or paranasal sinuses, 33 patients $(80.49 \%)$ with GPA and 12 (52.17\%) with MPA, respectively._32 patients $(50 \%)$ presented chronic rhinosinusitis (CRS). CRS was diagnosed in 26 patients (63.41\%) with GPA and in 6 patients (26.09\%) with MPA. 27 patients (42.18\%), 21 (51.22\%) with GPA and 6 (26.09\%) with MPA, suffered from recurrent, persistent epistaxis. These symptoms were the most frequently observed at the initial stage of the disease. We identified 10 cases of nasal septum perforation (NSP). NSP was found in 8 patients $(19.51 \%)$ with GPA and in 2 patients $(8.7 \%)$ with MPA. 22 patients $(34.38 \%)$ presented chronic purulent nasal discharge, 16 individuals (39.02\%) with GPA and 2 individuals $(8.7 \%)$ with MPA, respectively. Nasal ulcerations were identified in 15 patients $(23.43 \%), 12(29.27 \%)$ with GPA and 3 (13.04\%) with MPA.

Ears. The most frequent otologic disorders in our patients were sensorineural hearing loss and chronic otitis media followed by conductive hearing loss. Individuals with GPA were more prone to suffer from otologic dysfunction than those with MPA.

In our study, 36 patients $(56.25 \%)$ presented vasculitisrelated abnormalities in ears or in the sense of hearing, 25 patients $(60.98 \%)$ with GPA and $11(47.83 \%)$ with MPA, respectively. Hearing loss was identified in 32 patients (50\%), $24(58.54 \%)$ with GPA and $8(34.78 \%)$ with MPA. There were $18(28.13 \%)$ cases of sensorineural hearing loss, $12(18.75 \%)$ cases of conductive hearing loss, $8(12.5 \%)$ cases of mixed hearing loss, and 2 patients $(3.13 \%)$ with deafness identified in pure tone audiometry. Tinnitus was observed in 10 patients $(15.62 \%)$. Chronic otitis media was identified in 13 patients $(20.31 \%$ ), whereas otitis media with effusion was found in 8 patients (12.5\%). 4 individuals $(6.25 \%)$ suffered from recurrent episodes of purulent acute otitis media but without developing chronic otitis media.

Larynx. Laryngeal manifestations of AAV were not frequent in our study. Disorders in this localisation were found more often in patients with GPA than in those with MPA, except for laryngeal ulcerations that were more common in the latter group.

In a group of 64 enrolled patients, 12 (18.75\%) presented vasculitis-induced lesions in larynx. There were 8 patients $(12.5 \%)$ with GPA and $4(17.4 \%)$ with MPA with such involvement. Laryngeal abnormalities were not related to smoking status. Patients with positive smoking status were excluded from the study and statistics to minimalize the bias. 3 patients $(4.69 \%)$ presented subglottic stenosis, $2(4.88 \%)$ with GPA and 1 (4.35\%) with MPA. 2 patients with MPA and 1 patient with GPA were diagnosed with 
Table III. Clinical manifestations presenting organ involvement in specific types of AAV.

\begin{tabular}{|c|c|c|c|c|c|c|c|}
\hline $\begin{array}{l}\text { AAV } \\
\text { type organ involvement }\end{array}$ & $\begin{array}{c}\text { GPA } \\
n=41\end{array}$ & $\begin{array}{c}\text { MPA } \\
n=23\end{array}$ & $\begin{array}{c}\text { PR3-ANCA (+) } \\
n=34\end{array}$ & $\begin{array}{c}\text { MPO-ANCA (+) } \\
n=21\end{array}$ & $\begin{array}{c}\text { ANCA (-) } \\
n=7\end{array}$ & $\begin{array}{c}\text { MPO-ANCA (+) } \\
\text { and PR3-ANCA (+) n } \\
=2\end{array}$ & $\begin{array}{l}\text { Total } \\
n=64\end{array}$ \\
\hline ENT & 35 (85.37\%) & $\begin{array}{c}13 \\
(56.52 \%)\end{array}$ & $\begin{array}{c}29 \\
(85.29 \%)\end{array}$ & $\begin{array}{c}12 \\
(57.14 \%)\end{array}$ & $\begin{array}{c}5 \\
(71.43 \%)\end{array}$ & $\begin{array}{c}2 \\
(100 \%)\end{array}$ & $\begin{array}{c}48 \\
(75 \%)\end{array}$ \\
\hline Eyes & $\begin{array}{c}5 \\
(12.2 \%)\end{array}$ & $\begin{array}{c}3 \\
(13.04 \%)\end{array}$ & $\begin{array}{c}5 \\
(21.74 \%)\end{array}$ & $\begin{array}{c}3 \\
(14.29 \%)\end{array}$ & $\begin{array}{c}0 \\
(0 \%)\end{array}$ & $\begin{array}{c}0 \\
(0 \%)\end{array}$ & $\begin{array}{c}8 \\
(12.5 \%)\end{array}$ \\
\hline Lungs & $32(78.05 \%)$ & $\begin{array}{c}16 \\
(69.57 \%)\end{array}$ & $\begin{array}{c}29 \\
(85.29 \%)\end{array}$ & $\begin{array}{c}15 \\
(71.43 \%)\end{array}$ & $\begin{array}{c}3 \\
(42.86 \%)\end{array}$ & $\begin{array}{c}1 \\
(50 \%)\end{array}$ & $\begin{array}{c}49 \\
(76.56 \%)\end{array}$ \\
\hline Trachea & $\begin{array}{c}4 \\
(9.76 \%)\end{array}$ & $\begin{array}{c}1 \\
(4.35 \%)\end{array}$ & $\begin{array}{c}4 \\
(11.76 \%)\end{array}$ & $\begin{array}{c}1 \\
(4.76 \%)\end{array}$ & $\begin{array}{c}0 \\
(0 \%)\end{array}$ & $\begin{array}{c}0 \\
(0 \%)\end{array}$ & $\begin{array}{c}5 \\
(7.81 \%)\end{array}$ \\
\hline Kidneys & $26(63.41 \%)$ & $\begin{array}{c}21 \\
(91.3 \%)\end{array}$ & $\begin{array}{c}25 \\
(73.53 \%)\end{array}$ & $\begin{array}{c}19 \\
(90.48 \%)\end{array}$ & $\begin{array}{c}3 \\
(42.86 \%)\end{array}$ & $\begin{array}{c}0 \\
(0 \%)\end{array}$ & $\begin{array}{c}47 \\
(73.44 \%)\end{array}$ \\
\hline Cardiovascular system & $\begin{array}{c}8 \\
(19.51 \%)\end{array}$ & $\begin{array}{c}10 \\
(43.48 \%)\end{array}$ & $\begin{array}{c}8 \\
(23.53 \%)\end{array}$ & $\begin{array}{c}10 \\
(47.62 \%)\end{array}$ & $\begin{array}{c}0 \\
(0 \%)\end{array}$ & $\begin{array}{c}0 \\
(0 \%)\end{array}$ & $\begin{array}{c}18 \\
(28.13 \%)\end{array}$ \\
\hline Skin & $\begin{array}{c}12 \\
(29.27 \%)\end{array}$ & $\begin{array}{c}8 \\
(34.78 \%)\end{array}$ & $\begin{array}{c}11 \\
(32.35 \%)\end{array}$ & $\begin{array}{c}8 \\
(38.1 \%)\end{array}$ & $\begin{array}{c}1 \\
(14.26 \%)\end{array}$ & $\begin{array}{c}0 \\
(0 \%)\end{array}$ & $\begin{array}{c}20 \\
(31.25 \%)\end{array}$ \\
\hline $\begin{array}{l}\text { Gastrointestinal } \\
\text { system }\end{array}$ & $\begin{array}{c}2 \\
(4.88 \%)\end{array}$ & $\begin{array}{c}3 \\
(13.04 \%)\end{array}$ & $\begin{array}{c}2 \\
(5.88 \%)\end{array}$ & $\begin{array}{c}2 \\
(9.52 \%)\end{array}$ & $\begin{array}{c}1 \\
(14.26 \%)\end{array}$ & $\begin{array}{c}0 \\
(0 \%)\end{array}$ & $\begin{array}{c}5 \\
(7.81 \%)\end{array}$ \\
\hline $\begin{array}{l}\text { Musculoskeletal } \\
\text { system }\end{array}$ & 10 (24.39\%) & $\begin{array}{c}6 \\
(26.09 \%)\end{array}$ & $\begin{array}{c}10 \\
(29.41 \%)\end{array}$ & $\begin{array}{c}6 \\
(28.57 \%)\end{array}$ & $\begin{array}{c}0 \\
(0 \%)\end{array}$ & $\begin{array}{c}0 \\
(0 \%)\end{array}$ & $\begin{array}{c}16 \\
(25 \%)\end{array}$ \\
\hline Nervous system & $\begin{array}{c}8 \\
(19.51 \%)\end{array}$ & $\begin{array}{c}0 \\
(0 \%)\end{array}$ & $\begin{array}{c}8 \\
(23.53 \%)\end{array}$ & $\begin{array}{c}0 \\
(0 \%)\end{array}$ & $\begin{array}{c}0 \\
(0 \%)\end{array}$ & $\begin{array}{c}0 \\
(0 \%)\end{array}$ & $\begin{array}{c}8 \\
(12.5 \%)\end{array}$ \\
\hline
\end{tabular}

laryngeal ulcerations. Inspiratory dyspnoea was observed in 8 patients (12.5\%), 5 (12.2\%) with GPA and $3(13.0 \%)$ with MPA. 8 individuals (12.5\%), 7 (17.1\%) with GPA and $1(4.35 \%)$ with MPA, presented hoarseness. We identified 2 patients $(3.13 \%)$ with laryngeal oedema ( 2 individuals with GPA and no case related to MPA), 1 patient $(1.56 \%)$ with chronic laryngitis suffering from GPA and 1 patient $(1.56 \%)$ with aphonia and GPA. 2 patients $(3.13 \%)$ with GPA experienced peripheral unilateral vocal fold paralysis. There were no cases of supraglottic stenosis or laryngeal necrotising granuloma in our study.

Eyes. Ocular disorders were found in 8 patients $(12,5 \%)$. In general, the majority of ocular manifestations of AAV was identified in patients with GPA (3 patients; 13\%). Individuals with GPA (5 patients; $12.2 \%$ ) were less prone to suffer from ocular involvement in our research. The most common eye-related disorder was episcleritis. Episcleritis was observed in 5 patients $(7.8 \%), 3(13 \%)$ with MPA and 2 (4.9\%) with GPA, respectively. 3 individuals $(4.69 \%)$ from the study group, $2(4.8 \%)$ patients with GPA and $1(4.3 \%)$ with MPA, suffered from scleritis. Subconjuntival haemorrhages were identified in 3 patients $(4.69 \%)$ with a higher prevalence in patients with MPA. Sudden unilateral visual loss was found in 1 patient $(1.57 \%)$ diagnosed with GPA.

Unfortunately, there is a limitation of the data on ocular involvement because of the fact that only 36 patients
(56.25\%) were examined by an ophthalmologist. Accordingly, there could have been undiagnosed cases of ocular AAV-related abnormalities in our patients.

Vertigo. 6 patients $(9.38 \%), 3$ patients $(7.3 \%)$ with GPA and 3 patients (13.0\%) with MPA, experienced recurrent episodes of vertigo during vasculitis.

Oral cavity, oropharynx. We identified 4 cases (6.25\%) with ulcerations located in the oral cavity or oropharynx. The majority of cases was observed in patients with GPA. Salivary glands: Involvement of salivary glands was found only in patients with GPA. 2 patients $(3.12 \%)$ developed inflammation of the parotid gland, whereas 1 (1.57\%) experienced non-inflammatory enlargement of parotids.

\section{Lower respiratory tract involvement}

Lungs. Pulmonary involvement was a common presentation in the group of our patients. It was reported in 49 individuals $(76.56 \%), 32(78.04 \%)$ with GPA and 16 $(69.57 \%)$ with MPA, respectively. The most frequent disorder in the lower respiratory tract was pneumonia (29 cases; $45.31 \%$ ). Haemoptysis was reported in 18 individuals $(28.13 \%)$. 6 patients $(9.38 \%)$ suffered from recurrent bronchitis. Persistent cough was observed in 13 patients (20.31\%).

Trachea. Tracheal manifestations of AAV were found in a small part of our cohort. Only 5 patients $(7.81 \%)$ experienced abnormalities in this area, 4 patients $(9.8 \%)$ with 
GPA and 1 patient (4.3\%) with MPA. The majority of patients with tracheal involvement presented tracheitis (4 patients; $6.25 \%$ ). Tracheal necrotising granulomas were found in 1 patient $(1.59 \%)$ with GPA, similarly to tracheal stenosis (1 patient with GPA).

\section{Renal involvement}

AAV-related improper function of kidneys was detected in the vast majority of individuals. Patients with MPA were more prone to suffer from renal involvement than those with GPA.

Kidneys were affected in 47 patients (73.44\%), 26 $(63.41 \%)$ with GPA and 21 (91.3\%) with MPA. According to histopathologic findings, the majority of patients (10 patients) presented rapidly progressive glomerulonephritis (RPGN), which was the most common type of glomerulonephritis in our cohort. Additionally, we identified 3 patients with mesangial proliferative glomerulonephritis (MPGN) and 2 cases of focal segmental glomerulonephritis (FSGN).

In the group of patients with renal involvement, $31(66 \%)$ presented affection of ENT area, 20 patients $(42.6 \%)$ with GPA and 11 (23.4\%) with MPA. ENT manifestations preceded kidney dysfunction in all cases.

\section{Cardiovascular manifestations}

Cardiovascular disorders were identified in 18 patients $(28.13 \%)$ with predominance in patients with MPA. The most common presentation was hypertension (11 patients; $17.18 \%) .6$ individuals $(9.38 \%)$ suffered from valvular heart disease, $2(3.13 \%)$ were diagnosed with cardiomyopathy and $5(7.81 \%)$ presented a history of non-atherosclerotic ischaemic cardiac pain. Heart arrhythmias were reported in 4 cases $(6.25 \%)$.

\section{Cutaneous manifestations}

Vasculitis-induced cutaneous abnormalities occurred more often in patients with GPA than in those with MPA. We identified 20 individuals $(31.3 \%)$ with AAV-related cutaneous symptoms with purpura as the most common one (13 patients; 20.31\%). 4 individuals (6.25\%) developed skin ulcerations. Gangrene affected the fingers and toes, and was observed in 2 patients $(3.13 \%)$, one patient with GPA and one with MPA. Gangrene of fingers and toes was followed by their demarcation in both cases.

\section{Gastrointestinal tract manifestations}

Gastrointestinal tract was affected in 5 patients $(7.81 \%)$, 3 individuals (13\%) with MPA and 2 patients (4.88\%) with GPA. 3 patients (4.69\%), 2 patients $(4.88 \%)$ with GPA and $1(4.35 \%)$ with MPA, experienced gastrointestinal bleeding. In addition, 2 patients (3.13\%), 1 with GPA and 1 with MPA, developed intestinal ischaemia followed by partial necrosis of the small intestine. The necrosis identified in the small intestine required extensive excision. 1 patient $(1.56 \%)$ diagnosed with MPA and presenting ANCA negative status experienced infarct and rupture of the spleen. The same patient developed acute hepatic and spleen arteries dissections. The group with gastrointestinal involvement consisted of 2 subjects with PR3-ANCA positive antibodies, 2 subjects with positive MPO-ANCA and one patient with ANCA negative profile.

\section{Nervous system involvement}

We identified 8 individuals (12.5\%) suffering from AAVrelated nervous system disorders. All these patients presented GPA form of AAV with positive PR3-ANCA antibodies. Except for one patient, each of these 8 subjects presented ENT involvement. Otorhinolaryngological symptoms preceded nervous dysfunction in every case. We did not observe any nervous manifestations in patients with MPA.

We identified 4 cases of peripheral nervous system dysfunction, 3 cases of various cranial nerves palsies and 1 case of stroke. Peripheral nervous system dysfunction appeared as mononeuritis multiplex.

Cranial nerve palsy. Trigeminal nerve palsy was seen in 1 patient (1.57\%), facial nerve palsy in 2 patients $(3.13 \%)$, glossopharyngeal nerve palsy in 2 patients (3.13\%), vagal palsy in 2 patients $(3.13 \%)$, accessory nerve palsy in 1 patient $(1.57 \%)$ and hypoglossal nerve palsy in 1 patient $(1.57 \%)$. Vagal nerve palsy appeared as vocal fold paralysis (paralysis of vagus nerve branch called recurrent laryngeal nerve) in both cases.

1 patient presented bulbar palsy (dysfunction of IX, X, XI and XII cranial nerves).

\section{Musculoskeletal involvement}

Musculoskeletal system was affected in 16 patients (25\%), 10 patients $(24.4 \%)$ with GPA and $6(26.1 \%)$ with MPA, respectively. The main presentation in these individuals were recurrent arthritis and joints pain.

\section{Discussion}

The estimated annual incidences of GPA and MPA range from 2 to 12 cases per million population, with a prevalence of 23 to 160 cases per million population. While GPA is more frequent in Europe (especially in northern Europe) and United States than in Japan, MPA is more common in Japan and China than in Europe and United States ${ }^{15}$. In the majority of cases, GPA is characterised by 
the serum presence of PR3-ANCA antibodies and clinical involvement of organs forming "classic GPA triad". The "classic GPA triad" includes upper and lower respiratory tract affection with coexisting renal dysfunction ${ }^{16}$. Otorhinolaryngological manifestations are the initial symptoms of GPA in $80-95 \%$ of cases and usually precede its conversion to systemic disease ${ }^{17}$.

Patients with MPA typically present pulmonary abnormalities and renal dysfunction ${ }^{18}$. ENT involvement is not common in this type of vasculitis and is definitely rarer than in patients with GPA ${ }^{19}$. Sera taken from individuals with MPA frequently express MPO-ANCA antibodies ${ }^{20}$. The main aim of this study was to document clinicopathological features of patients with small-vessel vasculitis (GPA and MPA) referred to our university hospital between January 2016 and April 2017. We compared clinicopathological features observed in GPA patients with those seen in MPA individuals focusing on otorhinolaryngological disorders. We tried to understand if ENT involvement correlates with MPA because data on this matter is sparse and inconsistent. We focused on otorhinolaryngological manifestations, which are typically the initial symptoms of AAV (specially GPA) to emphasise the role of the otorhinolaryngologist in early diagnosis and disease management. It is well known that rapid diagnosis may significantly improve prognosis of patients with AAV, whereas delayed diagnosis significantly worsens prognosis.

In our study, the most commonly affected area was the lower respiratory tract (49 patients; $76.56 \%$ ), followed by ENT involvement (48 patients; 75\%). Pulmonary involvement typically appeared as pneumonia and haemoptysis. Similar to other studies, in our cohort the most common radiographic findings in lungs in GPA and MPA were nodules and inflammatory infiltrations ${ }^{21}{ }^{22}$. Kidney affection was also frequently observed in our cohort (47 subjects; $73.44 \%$ ). The most common renal disorder was RPGN, consistently with Chachar et al. ${ }^{23}$. Cutaneous involvement with purpura as the most frequent presentation was reported in 19 subjects $(31.25 \%)$. Cardiovascular manifestations were found in 18 patients $(28.13 \%)$. The majority of disorders in this group constituted hypertension and valvular heart disease. Musculoskeletal dysfunction appearing as recurrent arthritis and joints pain was detected in 16 individuals (25\%). Nervous system was affected in 8 cases $(12.5 \%)$ with mononeuritis multiplex reported in most. Similarly, ocular involvement was observed in 8 subjects (12.5\%) with episcleritis as the most common manifestation. The rarest area involved in our cohort (5 patients; $7.81 \%$ ) was gastrointestinal involvement with gastrointestinal bleeding as the most frequent manifesta- tion. Pulmonary, ENT and nervous involvements were more common in patients with GPA, whereas renal and gastrointestinal were mostly found in MPA, consistent with another study ${ }^{14}$. Inconsistent with one investigation ${ }^{13}$, but consistent with another ${ }^{13}$, our results revealed the greater prevalence of cutaneous, cardiovascular and ocular disorders in patients with MPA.

ENT manifestations detected in the study were present at the initial phase of AAV (in all cases with GPA and in several with MPA) and preceded AAV diagnosis. The mean duration since first ENT symptoms to AAV diagnosis in our patients reached 14.5 months, and was longer than reported by another author ${ }^{24}$. The overall prevalence of otorhinolaryngological abnormalities detected in the initial stage of AAV was $75 \%$. The presence of ENT symptoms in the early clinical picture reached $85.37 \%$ in GPA and $56.52 \%$ in MPA. In the group of 35 patients with GPA and coexisting ENT involvement, $29(82.86 \%)$ were positive for PR3-ANCA antibodies, 4 subjects (11.43\%) were ANCA negative and 2 (5.71\%) were positive for both PR3ANCA and MPO-ANCA. In the cohort of 13 patients with MPA and ENT involvement, 12 (92.3\%) were positive for MPO-ANCA, and 1 (7.69\%) was seronegative. According to our results, patients with localised SVV are more prone to be ANCA negative. The high prevalence of ENT manifestations in subjects with AAV was confirmed in various studies ${ }^{14}{ }^{25}$. Similar to another study, herein the localised form of AAV was more frequently observed in individuals with GPA than in those with MPA, whereas MPA rather than GPA predisposed to severe form of AAV ${ }^{14}$.

The most common disease manifestation in the head and neck area in both groups, GPA and MPA, was chronic rhinosinusitis followed by epistaxis and purulent nasal discharge. This observation confirmed that reported by Metaxaris et al. ${ }^{24}$. Similar to Ono et al., our study found that ears were also frequently affected with chronic otitis media and sensorineural hearing loss as the most frequent disorders ${ }^{26}$. Otitis media with effusion, conductive hearing loss and deafness were only found in patients with GPA. The majority of laryngeal disorders appeared as hoarseness (not related to smoking) and inspiratory dyspnoea. There were 3 cases of subglottic stenosis and no subject presenting supraglottic stenosis in our study. We observed 2 individuals with peripheral facial nerve palsy and 2 patients with vocal fold palsy.

In our cohort, GPA was more frequently observed in females than in males and in women the disease developed earlier, as in other studies ${ }^{13} 27$. The mean age at diagnosis reached 50.2 years and was similar to observed by other authors ${ }^{14}{ }^{25}$. The most commonly affected areas in patients with GPA were ENT, kidneys and lower respiratory tract, 
reaching $85.37 \%, 63.41 \%$ and $78.05 \%$, respectively. In 21 cases $(51.22 \%)$, localised GPA with otorhinolaryngological symptoms transformed to systemic disease, with renal and pulmonary involvement in the majority of patients. In contrast, in 14 subjects (34.15\%) otorhinolaryngological symptoms have not converted to systemic disease to date. Consistent with previous reports, in our study ENT involvement mainly appeared as chronic rhinosinusitis, purulent nasal discharge, epistaxis, chronic otitis media and sensorineural hearing loss, whereas pulmonary manifestations were mainly haemoptysis and pneumonia ${ }^{23} 25$. CBC typically revealed leukocytosis with neutrophilia, lymphopenia and anaemia, while urinalysis presented proteinuria with microscopic haematuria and gross haematuria in most cases. Increased CRP, ESR, serum creatinine and urea levels were also frequently observed. Moreover, we found that patients with GPA were more frequently colonised by $S$. aureus than individuals with MPA. Interestingly, the majority of patients with staphylococcal carriage presented a localised form of the disease.

Similar to other studies, our results show that MPA develops later than GPA ${ }^{1426}$. MPA was also more frequent in women than in men. Women developed MPA at younger age than men. For MPA, the most frequent manifestations were renal, pulmonary and ENT disorders reaching $91.3 \%, 69.57 \%$ and $56.52 \%$, respectively. Renal involvement in our patients with MPA was more common than in those with GPA. This observation was consistent with the results of other studies ${ }^{1328}$. Otorhinolaryngological abnormalities typically reported in this group were chronic rhinosinusitis, purulent nasal discharge, epistaxis and sensorineural hearing loss. Similar to GPA, CBC in patients with MPA also mainly revealed leukocytosis with neutrophilia, lymphopenia and anaemia, while urinalysis presented proteinuria with microscopic haematuria and gross haematuria in most cases. Nevertheless, CBC and urine abnormalities were more common in individuals with MPA. This same observation can also be made for serum creatinine and urea levels. In this study, increased serum creatinine levels were more frequently observed in patients with MPA than in those with GPA, consistent with other reports ${ }^{1314}$. In contrast to GPA, S. aureus colonisation in subjects with MPA was rare.

ANCA negative (seronegative) patients typically presented mainly otorhinolaryngological disorders, followed by pulmonary and renal dysfunctions. Significant ENT involvement in seronegative patients was also confirmed by other authors ${ }^{19}$. Five patients $(71.4 \%)$ in this group were diagnosed with GPA, among which 4 presented localised disease and one subject had a generalised form of disease with no ENT involvement. Two ANCA-negative subjects
(28.6\%) had MPA; in both cases it was a systemic form of AAV with ENT manifestations in only one subject. Consistent with another study, our seronegative patients were younger at diagnosis (46.1 years) in comparison to their ANCA positive counterparts and were more prone to present a limited form of disease ${ }^{19} 29$. ENT manifestations comprised epistaxis, chronic rhinosinusistis and otitis media, nasal septum perforation, saddle nose, nasal and oral ulcerations, hearing loss (conductive and sensorineural), inspiratory dyspnoea and hoarseness. Sinonasal symptoms were the most frequently observed. Pulmonary involvement appeared as haemoptysis, pneumonia and persistent cough. The most common laboratory finding was neutrophilia and lymphopenia in CBC. Microscopic haematuria and gross haematuria were reported in 3 patients. S. aureus colonisation was identified in 2 seronegative individuals with localised GPA. According to our results, we suggest that ANCA-negative patients might present a less systemic form of AAV. Interestingly, it was recently suggested that the presence of oral cavity ulceration, namely palate perforation could be a frequently observed not only in seronegative patients with GPA, but also in cocaine users ${ }^{30}$.

In contrast, subjects with positive status for both ANCA antibodies (PR3-ANCA and MPO-ANCA) expressed only a localised form of SVV limited to the upper respiratory tract and lungs. ENT disorders in this group comprised chronic rhinosinusitis, hearing loss (sensorineural and mixed) and laryngeal oedema. Pulmonary manifestations observed in one patient appeared as persistent cough and nodules detected in CT scan of the thoracic cavity. Laboratory abnormalities were detected in CBC (neutrophilia with lymphopenia) and ESR (increased level).

The proportion of organ involvement in particular forms of AAV is described below (Fig. 1, Table III). The results of our study emphasise that ENT involvement is a common presentation, especially in patients with GPA, but it is also observed in patients with MPA. Moreover,

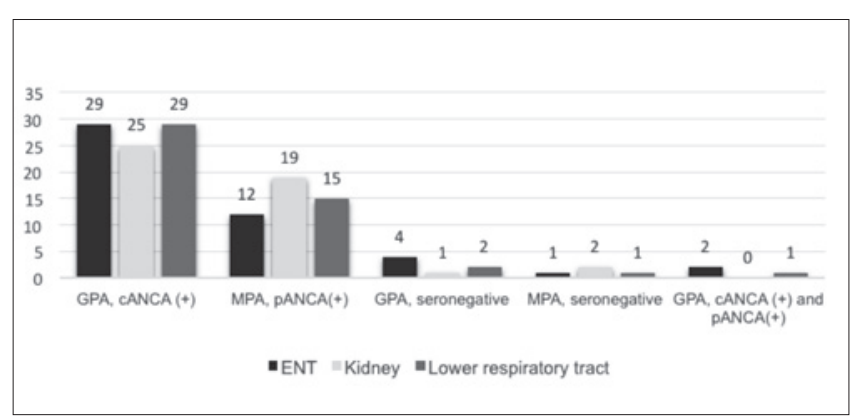

Fig. 1. Main organ involvement in various forms of AAV. 
ENT disorders typically precede SVV diagnosis and its transformation to systemic, frequently life-threatening, disease. Accordingly, we highlight the crucial role of the otorhinolaryngologist in early diagnosis of SVV.

Consistent with previously reported findings, according to our research, patients with GPA are more prone to present otorhinolaryngological disorders than those with MPA ${ }^{23}$. In contrast, the prevalence of renal and systemic dysfunctions (especially renal and pulmonary) is higher in subjects with MPA. Of great importance is the fact that ENT manifestations were the initial symptoms of AAV and preceded systemic organ disorders in the majority of cases. The average duration between first ENT symptoms and AAV diagnosis in our cohort was 14.5 months, ranging between 1 to 36 months. The most common initial ENT manifestations were recurrent epistaxis and persistent chronic rhinosinusitis not responding to standard treatment.

\section{Conclusions}

Our results highlight the significant role of the otorhinolaryngologist in early AAV diagnosis and management. We emphasise that any patient with persistent ENT symptoms or ENT dysfunctions (especially sinonasal) not responding to standard otorhinolaryngological treatment should be precisely and rapidly evaluated for the presence of systemic dysfunctions (especially renal and pulmonary). According to our observations, biochemical examination including CBC, ESR, CRP and PCT may hasten diagnosis. Evaluation of serum creatinine, serum urea and urinalysis are also strongly encouraged. Microbiological examination (towards Staphylococcal colonisation) should be carried out in patients with suspected AAV as well. ANCA presence tests are of great value in establishing AAV diagnosis, but negative results do not exclude the presence of disease. Interestingly, ANCA negative patients are prone to present a localised form of disease with only ENT manifestations. Thus, in such cases, the role of the otorhinolaryngologist is crucial. Early suspicion of SVV, proposed by an inquisitive otorhinolaryngologist, may lead to rapid disease diagnosis and prompt, adequate treatment. This could significantly improve the prognosis of this life-threatening disease.

\section{Conflict of interest statement}

None declared.

\section{References}

1 Castello E, Caligo G, Ravetti JL, et al. [Wegener's and Stewart's granulomatosis: a case report of Stewart's granulomatosis]. Acta Otorhinolaryngol Ital 1998;18:322-31.
2 Gomez-Puerta JA, Bosch X. Anti-neutrophil cytoplasmic antibody pathogenesis in small-vessel vasculitis: an update. Am J Pathol 2009; 175:1790-8.

3 Miller A, Chan M, Wiik A, et al. An approach to the diagnosis and management of systemic vasculitis. Clin Exp Immunol 2010;160:143-60.

4 Vacchi Suzzi M, Frasca G. [Clinical significance of "ANCA" in the diagnosis of Wegener's granulomatosis: 8 years of experience]. Acta Otorhinolaryngol Ital 1998;18:239-48.

5 Galli J, Corina L, Larocca LM. [Unusual case of pharyngeallaryngeal Wegener's granulomatosis]. Acta Otorhinolaryngol Ital 2001;21:187-91.

6 Srouji IA, Andrews P, Edwards C, et al. Patterns of presentation and diagnosis of patients with Wegener's granulomatosis: ENT aspects. J Laryngol Otol 2007;121:653-8.

7 Kuhn D, Hospowsky C, Both M, et al. Manifestation of granulomatosis with polyangiitis in head and neck. Clin Exp Rheumatol 2018;36(Suppl 111):78-84.

8 Gaffo AL. Diagnostic approach to ANCA-associated vasculitides. Rheum Dis Clin North Am 2010;36:491-506.

9 Stegeman CA, Tervaert JW, de Jong PE, et al. Trimethoprim-sulfamethoxazole (co-trimoxazole) for the prevention of relapses of Wegener's granulomatosis. Dutch Co-Trimoxazole Wegener Study Group. N Engl J Med 1996;335:16-20.

10 Leavitt RY, Fauci AS, Bloch DA, et al. The American College of Rheumatology 1990 criteria for the classification of Wegener's granulomatosis. Arthritis Rheum 1990;33:1101-7.

11 Khan I, Watts RA. Classification of ANCA-associated vasculitis. Curr Rheumatol Rep 2013;15:383.

12 Hellmich B, Flossmann O, Gross WL, et al. EULAR recommendations for conducting clinical studies and/or clinical trials in systemic vasculitis: focus on anti-neutrophil cytoplasm antibody-associated vasculitis. Ann Rheum Dis 2007;66:605-17.

13 Sada KE, Yamamura M, Harigai M, et al. Classification and characteristics of Japanese patients with antineutrophil cytoplasmic antibody-associated vasculitis in a nationwide, prospective, inception cohort study. Arthritis Res Ther 2014;16:R101.

14 Mahr A, Katsahian S, Varet H, et al. Revisiting the classification of clinical phenotypes of anti-neutrophil cytoplasmic antibody-associated vasculitis: a cluster analysis. Ann Rheum Dis 2013;72:1003-10.

15 Mohammad AJ, Jacobsson LT, Westman KW, et al. Incidence and survival rates in Wegener's granulomatosis, microscopic polyangiitis, Churg-Strauss syndrome and polyarteritis nodosa. Rheumatology (Oxford) 2009;48:1560-5.

16 Trimarchi M, Sinico RA, Teggi R, et al. Otorhinolaryngological manifestations in granulomatosis with polyangiitis (Wegener's). Autoimmun Rev 2013;12:501-5.

17 Wojciechowska J, Krajewski W, Krajewski P, et al. Granulomatosis with polyangiitis in otolaryngologist practice: 
a review of current knowledge. Clin Exp Otorhinolaryngol 2016;9:8-13.

18 Sinico RA, Di Toma L, Radice A. Renal involvement in antineutrophil cytoplasmic autoantibody associated vasculitis. Autoimmun Rev 2013;12:477-82.

19 Yoo J, Kim HJ, Ahn SS, et al. Clinical and prognostic features of Korean patients with MPO-ANCA, PR3-ANCA and ANCA-negative vasculitis. Clin Exp Rheumatol 2017;35(Suppl 103):111-8.

20 Greco A, De Virgilio A, Rizzo MI, et al. Microscopic polyangiitis: Advances in diagnostic and therapeutic approaches. Autoimmun Rev 2015;14:837-44.

21 Gomez-Gomez A, Martinez-Martinez MU, Cuevas-Orta E, et al. Pulmonary manifestations of granulomatosis with polyangiitis. Reumatol Clin 2014;10:288-93.

22 Tashiro H, Takahashi K, Tanaka M, et al. Characteristics and prognosis of microscopic polyangiitis with bronchiectasis. J Thorac Dis 2017;9:303-9.

23 Chachar AZ, Sabir O, Haider I, et al. Pulmonary renal syndrome in a patient with vasculitis: Case report and review of literature. Pak J Med Sci 2015;31:1545-8.

24 Metaxaris G, Prokopakis EP, Karatzanis AD, et al. Otolar- yngologic manifestations of small vessel vasculitis. Auris Nasus Larynx 2002;29:353-6.

25 Morales-Angulo C, Garcia-Zornoza R, Obeso-Aguera S, et al. [Ear, nose and throat manifestations of Wegener's granulomatosis (granulomatosis with polyangiitis)]. Acta Otorrinolaringol Esp 2012;63:206-11.

26 Ono N, Niiro H, Ueda A, et al. Characteristics of MPO-ANCA-positive granulomatosis with polyangiitis: a retrospective multi-center study in Japan. Rheumatol Int 2015;35:555-9.

27 Tsuchida Y, Shibuya M, Shoda H, et al. Characteristics of granulomatosis with polyangiitis patients in Japan. Mod Rheumatol 2015;25:219-23.

28 Hauer HA, Bajema IM, van Houwelingen HC, et al. Renal histology in ANCA-associated vasculitis: differences between diagnostic and serologic subgroups. Kidney Int 2002;61:80-9.

29 Cornec D, Cornec-Le Gall E, Fervenza FC, et al. $A N$ $C A$-associated vasculitis - clinical utility of using $A N$ CA specificity to classify patients. Nat Rev Rheumatol 2016;12:570-9.

30 Trimarchi M, Bondi S, Della Torre E, et al. Palate perforation differentiates cocaine-induced midline destructive lesions from granulomatosis with polyangiitis. Acta Otorhinolaryngol Ital 2017;374:281-5.

Address for correspondence: Joanna Wojciechowska, Department and Clinic of Otolaryngology, Head and Neck Surgery, Wrocław Medical University, Borowska 213, 50-556 Wrocław, Poland. Tel. +71 73437 00. E-mail wojciechowska.joan@gmail.com 